\title{
CORRECTION TERMS AND THE NON-ORIENTABLE SLICE GENUS
}

\author{
MARCO GOLLA AND MARCO MARENGON
}

\begin{abstract}
By considering negative surgeries on a knot $K$ in $S^{3}$, we derive a lower bound to the non-orientable slice genus $\gamma_{4}(K)$ in terms of the signature $\sigma(K)$ and the concordance invariants $V_{i}(\bar{K})$, which strengthens a previous bound given by Batson, and which coincides with OzsváthStipsicz-Szabó's bound in terms of their $v$ invariant for L-space knots and quasi-alternating knots. A curious feature of our bound is superadditivity, implying, for instance, that the bound on the stable non-orientable genus is sometimes better than the one on $\gamma_{4}(K)$.
\end{abstract}

\section{INTRODUCTION}

Given a knot $K$ in $S^{3}$, it is a very classical problem to determine the minimal genus of an orientable surface $F$ in $B^{4}$ whose boundary is $K$. More recently, some attention has been drawn to the case of non-orientable surfaces instead. Namely, one can define $\gamma_{4}(K)$ as the minimal non-orientable genus among all such surfaces, where the non-orientable genus of $F$ is defined as $b_{1}(F)$.

Batson, and Ozsváth, Stipsicz, and Szabó, on the other hand, gave lower bounds in terms of Heegaard Floer data. More precisely, Batson proved that

$$
\gamma_{4}(K) \geq \frac{\sigma(K)}{2}-d\left(S_{-1}^{3}(K)\right)
$$

where $d\left(S_{-1}^{3}(K)\right)$ is the Heegaard Floer correction term (or $d$-invariant) of the 3-manifold obtained as $(-1)$-surgery along $K$, in its unique $\operatorname{spin}^{c}$ structure (which is hence omitted from the notation) [2]. Ozsváth, Szabó and Stipsicz proved that

$$
\gamma_{4}(K) \geq\left|\frac{\sigma(K)}{2}-v(K)\right|,
$$

where $v$ is a concordance invariant defined in terms of the Floer homology package [18, Theorem 1.2]. Gilmer and Livingston gave lower bounds on $\gamma_{4}$ using Casson-Gordon invariants [11].

The main goal of this manuscript is to provide a new lower bound that generalises Batson's. It will be phrased in terms of the concordance invariants $\left\{V_{i}(\bar{K})\right\}_{i}$ associated to the mirror $\bar{K}$ of $K$; these invariants were defined by Rasmussen [21] and further studied by Ni and Wu [16] (see also Section 2 ] below). We will further package these invariants into a single integer-valued invariant that we call $\varphi$, $\varphi(K)=\min _{m \geq 0}\left\{m+2 V_{m}(\bar{K})\right\}$.

Theorem 1.1. For every knot $K$ in $S^{3}$,

$$
\gamma_{4}(K) \geq \frac{\sigma(K)}{2}-\varphi(K)
$$

The existence of such a bound was indicated, but not made explicit, by Batson in his PhD thesis [3]. Moreover, since $d\left(S_{-1}^{3}(K)\right)=2 V_{0}(\bar{K}) \geq \varphi(K)$, this is a strengthening of (1.1). Equation (1.3) also implies the existence of a bound in terms of the invariant $\nu^{+}$defined by Hom and Wu [12]. By definition, one has $V_{\nu^{+}(\bar{K})}(\bar{K})=0$, so Theorem 1.1 implies at once

$$
\gamma_{4}(K) \geq \frac{\sigma(K)}{2}-\nu^{+}(\bar{K}) .
$$

MG is supported by the Alice and Knut Wallenberg foundation.

MM was supported by an EPSRC Doctoral Training Award. 
Note that this bound is formally identical to (1.1), (1.2) and (1.3); to the best of the authors' knowledge, this bound never appeared in literature.

We will show below that the bound of Theorem 1.1 is sharp (see Remark 5.2), and agrees with the one of (1.2) in the case of alternating knots and L-space knots (see Proposition 6.2).

We note here that the bound (1.3) presents the following curious feature: it is superadditive in the knot $K$, in the sense that the bound for $K_{1} \# K_{2}$ can be strictly larger than the sum of the two bounds for $K_{1}$ and $K_{2}$. As a special case, the bound for $n K$ can give more information on $\gamma_{4}(K)$ than the bound for $K$. In Proposition 7.1 we will exhibit an example where this phenomenon actually occurs.

Using superadditivity, we can optimise the bound above as follows:

$$
\gamma_{4}(K) \geq \frac{\sigma(K)}{2}-\omega(K)
$$

where $\omega(K)$ is defined as

$$
\lim _{n \rightarrow \infty} \frac{1}{n} \varphi(n K) \leq \varphi(K) .
$$

Organisation of the paper. In Section 2 we recall some basic facts about $\operatorname{spin}^{c}$ structures on 3- and 4 -manifold and $d$-invariants, and we state all the results concerning them that we use in this paper. In Section 3 we fix the notation and we construct a cobordism $W^{\circ}$ from a particular 3-manifold $Q$ (defined in that section) to $S_{-n}^{3}(K)$, which will be crucial to deduce the bound in Equation (1.3). In Section 4 we label $\operatorname{spin}^{c}$ structures on $W^{\circ}$, compute their Chern classes, and understand their restrictions to $\partial W^{\circ}$. In Section 5 we apply a twisted version of Ozsváth-Szabó's inequality (see Theorem 2.4) to $W^{\circ}$ to obtain the desired bound on $\gamma_{4}(K)$. In Section 6 we compare our bound to Batson's and OzsváthStipsicz-Szabó's (see Equations (1.1) and (1.2)), and we refine it using superadditivity. Finally, in Section 7 we give an example of a knot $K$ where the bound for $n K$ is actually better the bound for $K$.

Acknowledgments. We would like to thank Tom Hockenhull for his encouragement and his patience; Antonio Alfieri, Fyodor Gainullin, Jen Hom, David Krcatovich, and András Stipsicz for interesting conversations; a special thanks goes to Joshua Batson for sharing some of his unpublished computations.

\section{All yOU NEED IS CORRECTION TERMS}

Given an oriented manifold $M$ of dimension 3 or 4 , recall that the set of $\operatorname{spin}^{c} \operatorname{structures}^{\operatorname{Spin}^{c}}(M)$ is an affine space over $H^{2}(M ; \mathbb{Z})$. Given an oriented 4 -manifold $X$ with boundary $\partial X=Y$, the restriction map

$$
\operatorname{Spin}^{c}(X) \rightarrow \operatorname{Spin}^{c}(Y)
$$

is modelled over

$$
H^{2}(X ; \mathbb{Z}) \rightarrow H^{2}(Y ; \mathbb{Z})
$$

To every $\operatorname{spin}^{c}$ structure $\mathfrak{s} \in \operatorname{Spin}^{c}(M)$ it is possible to associate an element in $H^{2}(M ; \mathbb{Z})$, known as the (first) Chern class of $\mathfrak{s}$, and usually denoted by $c_{1}(\mathfrak{s})$. The map

$$
c_{1}: \operatorname{Spin}^{c}(M) \rightarrow H^{2}(M ; \mathbb{Z})
$$

is injective if and only if $H^{2}(M ; \mathbb{Z})$ has no 2-torsion. A $\operatorname{spin}^{c} \operatorname{structure} \mathfrak{s} \in \operatorname{Spin}^{c}(M)$ is called torsion if $c_{1}(\mathfrak{s})$ is a torsion element in $H^{2}(M ; \mathbb{Z})$.

Let $-M$ denote the manifold $M$ endowed with the opposite orientation. There is a canonical bijection

$$
\iota: \operatorname{Spin}^{c}(M) \rightarrow \operatorname{Spin}^{c}(-M),
$$

which is modelled over the canonical isomorphism $\iota: H^{2}(M ; \mathbb{Z}) \rightarrow H^{2}(-M ; \mathbb{Z})$ (see [9, Section 1.2.3]). If $\mathfrak{s} \in \operatorname{Spin}^{c}(M)$, we will denote by the same letter $\mathfrak{s}$ the corresponding $\operatorname{spin}^{c}$ structure on $-M$. It is worth noting that such a bijection commutes with the restriction map (see Equation (2.1)), and that

$$
c_{1}(\iota(\mathfrak{s}))=\iota\left(c_{1}(\mathfrak{s})\right)
$$


Remark 2.1. Let $X^{4}$ be the trace of the 2-handle cobordism from $S^{3}$ to $S_{n}^{3}(K)$, where $K$ is a knot in $S^{3}$ and $n>0$ is a positive integer. Then we can label the $\operatorname{spin}^{c}$ structures on $X$ as follows: we let $\mathfrak{s}_{k}$ denote the unique $\operatorname{spin}^{c}$ structure on $X$ such that

$$
\left\langle c_{1}\left(\mathfrak{s}_{k}\right),[\Sigma]\right\rangle=n+2 k,
$$

where $\Sigma$ is a Seifert surface for $K$ in $S^{3} \times I$, capped off with the core of the 2-handle. From the labelling above, we derive a labelling of $\operatorname{spin}^{c}$ structures over $S_{n}^{3}(K)$ by $\mathbb{Z} / n \mathbb{Z}$, by setting

$$
\mathfrak{t}_{k}:=\left.\mathfrak{s}_{k}\right|_{S_{n}^{3}(K)},
$$

where we do not make the distinction between an integer and its class modulo $n$. Here and in the following, we refer the reader to [20, Section 2.4] for further details.

In what follows, we say that a pair $(Y, \mathfrak{t})$ as above, where $\mathfrak{t}$ is a torsion $\operatorname{spin}^{c}$ structure on the 3 -manifold $Y$, is a torsion $\operatorname{spin}^{c} 3$-manifold.

In [19, Ozsváth and Szabó introduce a Heegaard Floer theoretical invariant $d(Y, \mathfrak{t})$, called the correction term or d-invariant, associated to a pair $(Y, \mathfrak{t})$, where $Y$ is a rational homology 3 -sphere equipped with a $\operatorname{spin}^{c}$ structure $\mathfrak{t}$. In [19, Section 9], they explain how it is possible to generalise it to invariants $d_{b}$ and $d_{t}$ (bottom and top) associated to a torsion $\operatorname{spin}^{c} 3$-manifold $(Y, \mathfrak{t}$ ), where $Y$ is now a 3-manifold with standard $\mathrm{HF}^{\infty}$ (which is equivalent to having trivial triple cup product 15]). See also [14, Section 3] for an introduction to $d$-invariants of arbitrary 3 -manifolds with standard $\mathrm{HF}^{\infty}$. Behrens and the first author used Heegaard Floer homology with twisted coefficients to generalise this further to an invariant $\underline{d}(Y, \mathfrak{t})$ associated to an arbitrary torsion $\operatorname{spin}^{c} 3$-manifold $(Y, \mathfrak{t})$ [ $]$.

In the case of rational homology 3 -spheres we have

$$
d(Y, \mathfrak{t})=d_{b}(Y, \mathfrak{t})=d_{t}(Y, \mathfrak{t})=\underline{d}(Y, \mathfrak{t}) .
$$

More generally, one has the following.

Theorem 2.2 ([19, Proposition 4.2], [14, Proposition 3.7], and [4, Proposition 3.8]). Let (Y, t) be a torsion spin ${ }^{c} 3$-manifold, and suppose that $Y$ has standard $\mathrm{HF}^{\infty}$. Then, under the canonical identification $\operatorname{Spin}^{c}(Y) \cong \operatorname{Spin}^{c}(-Y)$,

$$
d_{b}(Y, \mathfrak{t})=-d_{t}(-Y, \mathfrak{t})=\underline{d}(Y, \mathfrak{t}) .
$$

In the rest of this section, we state the results that we need about $d$-invariants.

The following result by $\mathrm{Ni}$ and $\mathrm{Wu}$ allows us to compute $d$-invariants for surgeries on a knot $K \subseteq S^{3}$ in terms of some knot invariants $V_{i}$, which were first introduced in [21] with the name of $h_{i}$. We refer to [16, Section 2.2] for the definition of $V_{i}$.

Theorem 2.3 ([16, Proposition 1.6 and Remark 2.10]). Given positive integers $0 \leq k<n$, then

$$
d\left(S_{n}^{3}(K), \mathfrak{t}_{k}\right)=-\frac{n-(2 k-n)^{2}}{4 n}-2 \max \left\{V_{k}(K), V_{n-k}(K)\right\} .
$$

Correction terms can be used to give restrictions to intersection forms of 4-manifolds bounding a given 3-manifold (compare also with [19, Theorem 9.15]).

Theorem 2.4 (4, Theorem 4.1]). Let $(W, \mathfrak{s})$ be a negative semi-definite $\operatorname{spin}^{c}$ cobordism from $(Y, \mathfrak{t})$ to $\left(Y^{\prime}, \mathfrak{t}^{\prime}\right)$, two torsion spin ${ }^{c} 3$-manifolds, such that the map $H_{1}(Y ; \mathbb{Q}) \rightarrow H_{1}(W ; \mathbb{Q})$ induced by the inclusion is injective. Then

$$
c_{1}(\mathfrak{s})^{2}+b_{2}^{-}(X) \leq 4 \underline{d}\left(Y^{\prime}, \mathfrak{t}^{\prime}\right)+2 b_{1}\left(Y^{\prime}\right)-4 \underline{d}(Y, \mathfrak{t})-2 b_{1}(Y) .
$$

\section{Notation AND CONSTRUCTION}

Let $K$ be a knot in $S^{3}$. If we consider $S^{3}$ as the boundary of the 4-ball $B^{4}$, the (orientable) slice genus $g_{4}$ is defined as the minimum genus of a smooth orientable surface is $B^{4}$ whose boundary is $K$, and it is a well-studied invariant of $K$. More recently, the non-orientable slice genus $\gamma_{4}$ has been studied. We have the following definition. 


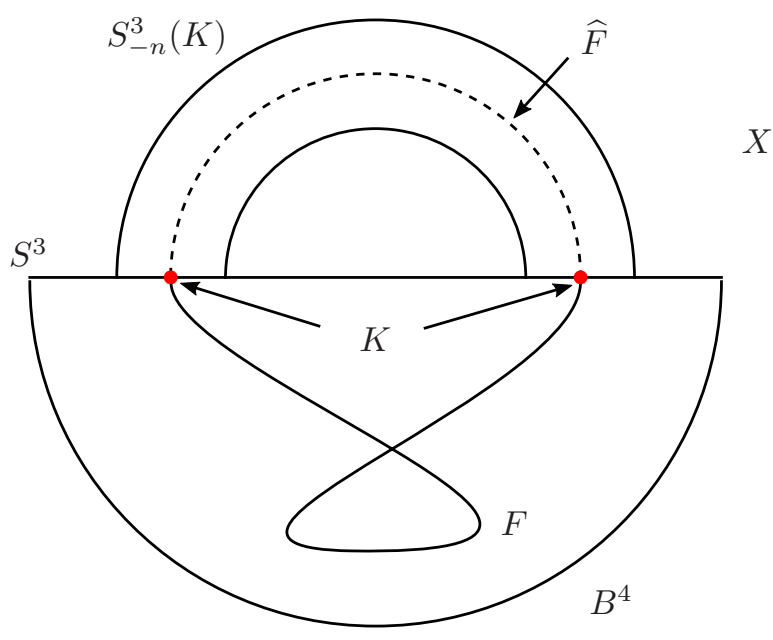

Figure 1. The figure shows the 4-manifold $W$ obtained by attaching a $(-n)$-framed 2-handle (whose trace we denote by $X)$ to $B^{4}$ along a knot $K \subseteq S^{3} . N=\mathcal{N}_{W}(\widehat{F})$ denotes a neighbourhood of $\widehat{F}$ in $W$, and $Q=\partial N$.

Definition 3.1. Given a knot $K$ in $S^{3}$, we define its non-orientable slice genus as

$$
\gamma_{4}(K)=\min \left\{b_{1}(F) \mid F \hookrightarrow B^{4} \text { smooth, non-orientable, } \partial F=K\right\},
$$

where $b_{1}(F)$ denotes the first Betti number of $F$.

Remark 3.2. With this definition of $\gamma_{4}$, one always has $\gamma_{4}(K) \geq 1$. One could also consider the 4dimensional crosscap number instead; this is the minimal number $h$ such that $K$ bounds a punctured $\#^{h} \mathbb{R P}^{2}$ in $B^{4}$. The two definitions are indeed equivalent except when $K$ is slice, in which case our definition yields $\gamma_{4}(K)=1$, while the 4 -dimensional crosscap number is 0 . We note here that, when $K$ is slice, the bound in (1.3) is in any case $\gamma_{4}(K) \geq 0$, so this is in fact a bound for the crosscap number as well; this is true since, when $K$ is slice, both $\sigma(K)$ and $\varphi(L)$ vanish (see Proposition 6.1 (2) below). Our proof, however, actually uses the definition of $\gamma_{4}$ given above, to which therefore we stick.

In 2, Batson proved that the non-orientable slice genus can be arbitrarily large. More specifically, for a non-orientable surface $F$ as in Definition 3.1. Batson gives the following inequality (see 2, Theorem 4]):

$$
b_{1}(F)+2 d\left(S_{-1}^{3}(K)\right) \geq \frac{e(F)}{2} .
$$

Here $d\left(S_{-1}^{3}(K)\right)$ denotes the $d$-invariant of $S_{-1}^{3}(K)$ in the unique $\operatorname{spin}^{c} \operatorname{structure}$, whereas $e(F)$ is the normal Euler number of $F$ : given a non-vanishing section $s$ of the normal bundle $\nu_{F}$ (which always exists since $F$ deformation retracts on a 1 -complex), we let

$$
e(F)=-\operatorname{lk}(K, s(K)) .
$$

In [2, Batson combines Equation (3.1) and the 'signature' inequality

$$
b_{1}(F) \geq \sigma(K)-\frac{e(F)}{2}
$$

to derive the bound for the non-orientable slice genus in Equation (1.1). The main result of this paper is a generalisation of Equation (3.1), where instead of the (-1)-surgery along $K$ we consider $(-n)$ surgeries for arbitrary integers $n \geq 1$. Inspired by [2] and 14, we construct a negative semi-definite cobordism from a 3-manifold $Q$ to $S_{-n}^{3}(K)$, and use Theorem 2.4 to give a lower bound to $b_{1}(F)$.

We now give the details of the construction, illustrated in Figure 1, Let $K$ be a knot in $S^{3}=\partial B^{4}$, and let $F$ denote a smooth non-orientable surface properly embedded in $B^{4}$ such that $\partial F=K$. Fix 
an integer $n>0$. Let $W$ denote the 4-manifold obtained by attaching a $(-n)$-framed 2-handle to $B^{4}$, along $K \subset \partial B^{4}$. We denote with $Y$ the boundary of $W$, i.e. $Y=S_{-n}^{3}(K)$. Then the surface $F$ can be capped off with the core of the 2-handle to obtain a closed surface $\widehat{F} \subseteq W$. Notice that

$$
b_{1}(\widehat{F})+1=b_{1}(F)=: h .
$$

If $e=e(F)$ denotes the normal Euler number of $F$, and $e(\widehat{F})$ denotes the Euler number of the closed surface $\widehat{F}$, then we have

$$
e(\widehat{F})=e-n \text {. }
$$

As already noticed in [2], $e$ is even, because the self-intersection of $F$ in $B^{4}$ can be computed algebraically over $\mathbb{Z} / 2 \mathbb{Z}$.

Let $N=\mathcal{N}_{W}(\widehat{F})$ denote a regular neighbourhood of $\widehat{F}$ in $W$. We define $Q=\partial N$. Notice that $Q$ (resp. $N$ ) is a circle (resp. disc) bundle over the closed surface $\widehat{F} \cong\left(\mathbb{R P}^{2}\right)^{\# h}$ of Euler number $e-n$. According to the notation in [14, Section 2], we have $N \cong P_{h, e-n}$ and $Q \cong Q_{h, e-n}$, and moreover $Q$ has standard $\mathrm{HF}^{\infty}$.

The manifold $W^{\circ}:=W \backslash N$ is a cobordism between $Q$ and $S_{-n}^{3}(K)$. Since the labelling of $\operatorname{spin}^{c}$ structures is better understood for positive surgeries, we consider also the manifold $-W$, obtained from $W$ by reversing the orientation; $-W$ is the 4 -manifold obtained by attaching an $n$-framed 2-handle to $B^{4}$ along $\bar{K}$. This allows us to label the $\operatorname{spin}^{c}$ structures on $W$ and on $Y$; by a slight abuse of notation, we write $\mathfrak{s}_{k}$ and $\mathfrak{t}_{k}$, dropping the identifications $\operatorname{Spin}^{c}(W) \cong \operatorname{Spin}^{c}(-W)$ and $\operatorname{Spin}^{c}(Y)=\operatorname{Spin}^{c}(-Y)$.

\section{LABELLING Spin ${ }^{c}$ STRUCTURES}

4.1. (Co-)homological computations. The aim of this subsection is to compute $H^{2}\left(W^{\circ} ; \mathbb{Z}\right)$, in order to understand $\operatorname{spin}^{c}$ structures on $W^{\circ}$. Consider the Mayer-Vietoris long exact sequence in cohomology associated to $W=W^{\circ} \cup_{Q} N$. When we do not specify it, we assume that we are using $\mathbb{Z}$ coefficients.

\begin{tabular}{c||c|ccc|c} 
& $W$ & $W^{\circ}$ & $\sqcup$ & $N$ & $Q$ \\
\hline$H^{0}$ & $\mathbb{Z}$ & $\mathbb{Z}$ & $\oplus$ & $\mathbb{Z}$ & $\mathbb{Z}$ \\
$H^{1}$ & 0 & 0 & $\oplus$ & $\mathbb{Z}^{h-1}$ & $\mathbb{Z}^{h-1}$ \\
$H^{2}$ & $\mathbb{Z}$ & $?$ & $\oplus$ & $\mathbb{Z} / 2 \mathbb{Z}$ & $\mathbb{Z}^{h-1} \oplus T$ \\
$H^{3}$ & 0 & $\mathbb{Z}$ & $\oplus$ & 0 & $\mathbb{Z}$
\end{tabular}

The cohomology of $W$ can be easily obtained by recalling that $W$ is constructed by attaching a 2-handle on a $B^{4}$. The cohomology of $N$ is also straightforward, since $N$ deformation retracts on $\widehat{F}=\left(\mathbb{R P}^{2}\right)^{\# h}$. As for $Q$, its cohomology can be deduced from [14, Lemma 2.1], and it is written in the table above. $T$ is the torsion subgroup of $H_{1}(Q)$, which is, according to [14, Lemma 2.1],

$$
T= \begin{cases}\mathbb{Z} / 2 \mathbb{Z} \oplus \mathbb{Z} / 2 \mathbb{Z} & \text { if } e(\widehat{F}) \text { is even, } \\ \mathbb{Z} / 4 \mathbb{Z} & \text { if } e(\widehat{F}) \text { is odd. }\end{cases}
$$

In both cases, the map $H^{2}(N) \cong \mathbb{Z} / 2 \mathbb{Z} \rightarrow T$ is non-trivial. From the cohomology groups that we already know (and the fact that the map $H^{1}(N) \rightarrow H^{1}(Q)$ is an isomorphism) we can deduce almost all the cohomology groups of $W^{\circ} \cdot H^{2}\left(W^{\circ}\right)$ will depend on the parity of $e(\widehat{F})$, according to the following lemma.

Lemma 4.1. We have that

$$
H^{2}\left(W^{\circ}\right)= \begin{cases}\mathbb{Z}^{h} \oplus \mathbb{Z} / 2 \mathbb{Z} & \text { if } e(\widehat{F}) \text { is even }, \\ \mathbb{Z}^{h} & \text { if } e(\widehat{F}) \text { is odd. }\end{cases}
$$

Proof. From the long exact sequence above we have an exact sequence

$$
0 \rightarrow \mathbb{Z} \rightarrow H^{2}\left(W^{\circ}\right) \rightarrow \mathbb{Z}^{h-1} \oplus \mathbb{Z} / 2 \mathbb{Z} \rightarrow 0,
$$

regardless of the parity of $e(\widehat{F})$. The two possible extensions are $\mathbb{Z}^{h} \oplus \mathbb{Z} / 2 \mathbb{Z}$ and $\mathbb{Z}^{h}$. If we consider the reduction modulo 2 , which maps $H^{2}\left(W^{\circ}\right)$ to $H^{2}\left(W^{\circ} ; \mathbb{F}_{2}\right)$, the two possible extensions are mapped 
respectively to $\mathbb{F}_{2}^{h+1}$ and $\mathbb{F}_{2}^{h}$. Therefore, in order to understand $H^{2}\left(W^{\circ}\right)$, it is enough to determine the rank of $H^{2}\left(W^{\circ} ; \mathbb{F}_{2}\right)$.

Consider the Mayer-Vietoris long exact sequence in homology associated to $W=W^{\circ} \cup_{Q} N$, with $\mathbb{F}_{2}$ coefficients. Since the coefficient ring is a field, homology and cohomology are dual to each other. As in the previous case, the homologies of $W$ and $N$ are quite straightforward to compute. According to 14 , Proof of Lemma 2.1], $H_{1}\left(Q ; \mathbb{F}_{2}\right) \cong H_{2}\left(Q ; \mathbb{F}_{2}\right) \cong \mathbb{F}_{2}^{h+1}$ if $e(\widehat{F})$ is even, and $H_{1}\left(Q ; \mathbb{F}_{2}\right) \cong H_{2}\left(Q ; \mathbb{F}_{2}\right) \cong \mathbb{F}_{2}^{h}$ if $e(\widehat{F})$ is odd.

\begin{tabular}{c||c|ccc|c} 
& $Q$ & $W^{\circ}$ & $\sqcup$ & $N$ & $W$ \\
\hline$H_{3}$ & $\mathbb{F}_{2}$ & $\mathbb{F}_{2}$ & $\oplus$ & 0 & 0 \\
$H_{2}$ & $H_{2}\left(Q ; \mathbb{F}_{2}\right)$ & $?$ & $\oplus$ & $\mathbb{F}_{2}$ & $\mathbb{F}_{2}$ \\
$H_{1}$ & $H_{1}\left(Q ; \mathbb{F}_{2}\right)$ & $?$ & $\oplus$ & $\mathbb{F}_{2}^{h}$ & 0 \\
$H_{0}$ & $\mathbb{F}_{2}$ & $\mathbb{F}_{2}$ & $\oplus$ & $\mathbb{F}_{2}$ & $\mathbb{F}_{2}$
\end{tabular}

Consider the connecting morphism

$$
\partial: H_{2}\left(W ; \mathbb{F}_{2}\right) \rightarrow H_{1}\left(Q ; \mathbb{F}_{2}\right) .
$$

If $\alpha=[\widehat{F}]$ is the generator of $H_{2}\left(W ; \mathbb{F}_{2}\right) \cong \mathbb{F}_{2}$ and $\gamma$ is the class of a circle fibre in $H_{1}\left(Q ; \mathbb{F}_{2}\right)$, then

$$
\partial \alpha=e(\widehat{F}) \cdot \gamma \in H_{1}\left(Q ; \mathbb{F}_{2}\right) .
$$

It follows that the map $\partial$ is trivial when $e(\widehat{F})$ is even.

The map $\partial: H_{2}\left(W ; \mathbb{F}_{2}\right) \rightarrow H_{1}\left(Q ; \mathbb{F}_{2}\right)$ is trivial also when $e(\widehat{F})$ is odd. This is because -according to [14, Lemma 2.1] — when $e(\widehat{F})$ is odd the homology class of the fibre is divisible by 2 in $H_{1}(Q)$, so its reduction modulo 2 is 0 .

From this we deduce that $H_{2}\left(W^{\circ} ; \mathbb{F}_{2}\right) \cong \mathbb{F}_{2}^{h}$ if $e(\widehat{F})$ is odd, and $H_{2}\left(W^{\circ} ; \mathbb{F}_{2}\right) \cong \mathbb{F}_{2}^{h+1}$ if $e(\widehat{F})$ is even, and hence we conclude the proof of the lemma.

4.2. Intersection form. In this section we study the intersection forms on $H_{2}(W)$ and $H^{2}(W)$.

Lemma 4.2. The intersection form $Q_{W}$ on $H_{2}(W) \cong \mathbb{Z}$ is given by $Q_{W}=(-n)$. The intersection form $Q^{W}$ on $H^{2}(W) \cong \mathbb{Z}$ is given by $Q^{W}=\left(-\frac{1}{n}\right)$.

Proof. The intersection form on $H_{2}(W)$ is $(-n)$ because the 4-manifold $W$ is obtained by attaching a $(-n)$-framed 2-handle to $B^{4}$.

The intersection form on $H^{2}(W)$ can be worked out by considering the following portion of the long exact sequence in homology associated to the couple $(W, Y)$, where $Y=S_{-n}^{3}(K)$ :

$$
0 \rightarrow H_{2}(W) \rightarrow H^{2}(W) \rightarrow H_{1}(Y) \rightarrow 0
$$

Such a short exact sequence is isomorphic to

$$
0 \rightarrow \mathbb{Z} \rightarrow \mathbb{Z} \rightarrow \mathbb{Z} / n \mathbb{Z} \rightarrow 0
$$

The generator of $H_{2}(W)$ is mapped to $n$ times the generator of $H^{2}(W)$, so the intersection form on $H^{2}(W)$ is represented by the matrix $\left(-\frac{1}{n}\right)$.

It is also worth noting that for each $c \in H^{2}(W),\left.c\right|_{W^{\circ}}$ restricts to a torsion spin $^{c}$ structure on both boundary components, and therefore it makes sense to consider its square. We claim that:

$$
Q^{W^{\circ}}\left(\left.c\right|_{W^{\circ}}\right)=Q^{W}(c)
$$

Indeed, the class $n c \in H^{2}(W) \cong H_{2}(W, Y)$ is in the image of the map $H_{2}(W) \rightarrow H_{2}(W, Y)$. Now, the map $\iota: H_{2}\left(W^{\circ}\right) \rightarrow H_{2}(W)$ is surjective: this comes from the Mayer-Vietoris sequence for $W=W^{\circ} \cup N$, since the connecting morphism $\partial: H_{2}(W) \rightarrow H_{1}(Q)$ vanishes (see the proof of Lemma 4.1) and $H_{2}(N)=0$.

Therefore, there is an element $d \in H_{2}\left(W^{\circ}\right)$ such that $\iota(d)=n c$. The elements $d$ and $n c$ can be represented by some copies of a surface $S \subseteq W^{\circ}$. The squares $Q^{W^{\circ}}(d)$ and $Q^{W}(n c)$ can be computed as the algebraic self-intersection $S \cdot S$ of $S$, which in turn can be computed in an arbitrarily small neighbourhood of $S$. 
4.3. Spin ${ }^{c}$ structures. Recall (see Remark 2.1) that $\operatorname{spin}^{c}$ structures on $-W$ are labelled by integers as follows:

$$
\left\langle c_{1}\left(\mathfrak{s}_{k}\right),[\Sigma]\right\rangle=2 k+n .
$$

By symmetry we also get a labelling for $\operatorname{Spin}^{c}(W)$, and we still denote the $\operatorname{spin}^{c}$ structures on $W$ by $\mathfrak{s}_{k} . \mathfrak{s}_{k}$ and $\mathfrak{s}_{k^{\prime}}$ restrict to the same $\operatorname{spin}^{c}$ structure on $Y$ if and only if $n \mid\left(k-k^{\prime}\right)$. In such a case we denote the restriction to $Y$ by $\mathfrak{t}_{k}=\mathfrak{t}_{k^{\prime}}$.

It is worth noting that we have isomorphisms $H^{2}(W) \cong \mathbb{Z}$ and $H^{2}(Y) \cong \mathbb{Z} / n \mathbb{Z}$ such that, under these identifications, the restriction map is the usual projection $\mathbb{Z} \rightarrow \mathbb{Z} / n \mathbb{Z}$, and $c_{1}\left(\mathfrak{t}_{k}\right) \equiv 2 k(\bmod n)$.

In order to apply Theorem 2.4 we need a $\operatorname{spin}^{c}$ structure on the cobordism $W^{\circ}$ that restricts to a torsion $\operatorname{spin}^{c}$ structure on $Q$. Therefore, we introduce the following notation:

Definition 4.3. Given a 4-manifold $X$, we define $\operatorname{Spin}_{\text {tor }}^{c}(X)$ to be the subset of $\operatorname{Spin}^{c}(X)$ of elements that restrict to torsion $\operatorname{spin}^{c}$ structures on $\partial X$.

Notice that in our case $\operatorname{Spin}_{\text {tor }}^{c}\left(W^{\circ}\right)$ is given by all $\operatorname{spin}^{c}$ structures that restrict to torsion $\operatorname{spin}^{c}$ structures on $Q$, because all $\operatorname{spin}^{c}$ structures on $Y$ are already torsion. We will now give a classification of $\operatorname{Spin}_{\text {tor }}^{c}\left(W^{\circ}\right)$ in the case of $e(\widehat{F})$ odd (or, equivalently, $n$ odd).

4.4. The case $e(\widehat{F})$ odd. By Lemma 4.1 we have that $H^{2}\left(W^{\circ}\right) \cong \mathbb{Z}^{h}$. From the Mayer-Vietoris exact sequence associated to $W=W^{\circ} \cup_{Q} N$ we find:

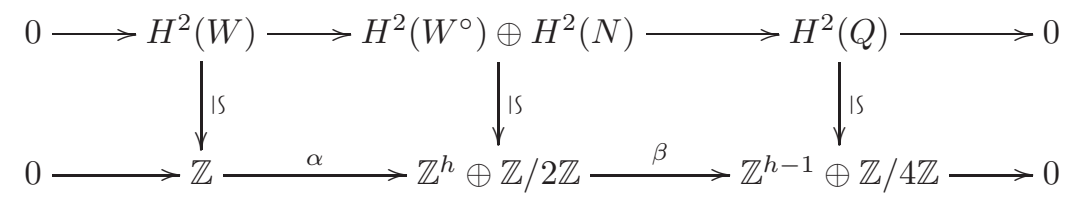

We have that $\alpha(1)=(c, 1)$ for some nonzero $c \in \mathbb{Z}^{h}$, otherwise the quotient would contain a $\mathbb{Z} / 2 \mathbb{Z}$ summand. Then we have that

$$
\mathbb{Z}^{h-1} \oplus \mathbb{Z} / 4 \mathbb{Z} \cong \frac{\mathbb{Z}^{h} \oplus \mathbb{Z} / 2 \mathbb{Z}}{\langle(c, 1)\rangle} \cong \frac{\mathbb{Z}^{h}}{\langle 2 c\rangle}
$$

This implies that $c=2 d$, where $d \in \mathbb{Z}^{h}$ is a primitive element. We denote by $x \in H^{2}\left(W^{\circ}\right)$ the element that corresponds to $d$, and we let $\mathcal{A}=\langle x\rangle \subseteq H^{2}\left(W^{\circ}\right)$. Therefore, $\operatorname{Spin}_{\text {tor }}^{c}\left(W^{\circ}\right)$ is an affine space over $\mathcal{A}$.

It follows from the exact sequence above that the image of the map

$$
\operatorname{Spin}^{c}(W) \rightarrow \operatorname{Spin}^{c}\left(W^{\circ}\right)
$$

is contained inside $\operatorname{Spin}_{\text {tor }}^{c}\left(W^{\circ}\right)$. Moreover, the map is modelled on the map

$$
H^{2}(W) \cong \mathbb{Z} \stackrel{\cdot 2}{\rightarrow} \mathbb{Z} \cong \mathcal{A} .
$$

It follows from the naturality of the first Chern class that $c_{1}\left(\left.\mathfrak{s}_{k}\right|_{W^{\circ}}\right)=(2 n+4 k) x$ :

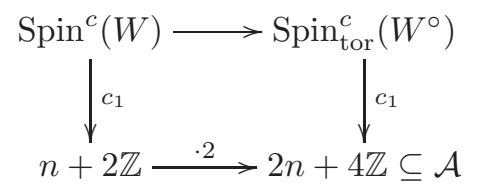

The Chern classes of all $\operatorname{spin}^{c}$ structures in $\operatorname{Spin}_{\text {tor }}^{c}\left(W^{\circ}\right)$ form the subset $2 n+2 \mathbb{Z}=2 \mathbb{Z} \subseteq \mathbb{Z} \cong \mathcal{A}$. This motivates the following definition.

Definition 4.4. We define $\mathfrak{s}_{k}^{\circ} \in \operatorname{Spin}_{\text {tor }}^{c}\left(W^{\circ}\right)$ to be the $\operatorname{spin}^{c}$ structure on $W^{\circ}$ that restricts to a torsion $\operatorname{spin}^{c}$ structure on $Q$ and that satisfies

$$
c_{1}\left(\mathfrak{s}_{k}^{\circ}\right)=(2 n+2 k) x .
$$


Remark 4.5. It follows from the computations above that

$$
\begin{aligned}
\operatorname{Spin}^{c}(W) & \rightarrow \operatorname{Spin}_{\text {tor }}^{c}\left(W^{\circ}\right) \\
\mathfrak{s}_{k} & \mapsto \mathfrak{s}_{2 k}^{\circ}
\end{aligned}
$$

and that $\mathfrak{s}_{k}^{\circ} \in \operatorname{Spin}_{\text {tor }}^{c}\left(W^{\circ}\right)$ extends to a $\operatorname{spin}^{c}$ structure on $W$ if and only if $k$ is even.

We now want to understand the restriction of the $\operatorname{spin}^{c}$ structure $\mathfrak{s}_{k}^{\circ}$ to $Y$. This is done in the following lemma. Instead of $W$, we use $W_{n}=-W$ and $S_{n}^{3}(\bar{K})=-Y$ to label the $\operatorname{spin}^{c}$ structure, so we can stick to the usual positive surgery conventions.

Lemma 4.6. For all $k \in \mathbb{Z}$ we have that

$$
\left.\mathfrak{s}_{2 k}^{\circ}\right|_{S_{n}^{3}(\bar{K})}=\mathfrak{t}_{k} \text { and }\left.\mathfrak{s}_{n+2 k}^{\circ}\right|_{S_{n}^{3}(\bar{K})}=\mathfrak{t}_{k} .
$$

Proof. Consider the following commutative diagram:
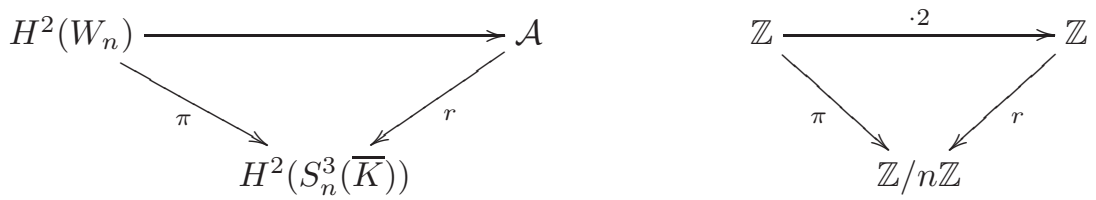

Recall that we chose isomorphisms $H^{2}\left(W_{n}\right) \cong \mathbb{Z}$ and $H^{2}\left(S_{n}^{3}(\bar{K})\right) \cong \mathbb{Z} / n \mathbb{Z}$ such that $\pi(1)=1 \in \mathbb{Z} / n \mathbb{Z}$. Then

$$
c_{1}\left(\mathfrak{t}_{k}\right)=\pi\left(c_{1}\left(\mathfrak{s}_{k}\right)\right)=n+2 k=2 k .
$$

Since $n$ is odd, 2 is invertible modulo $n$, so every $\operatorname{spin}^{c}$ structure on $S_{n}^{3}(\bar{K})$ is determined by its first Chern class.

By the naturality of the Chern class we have that for every $k \in \mathbb{Z}$, the following diagram commutes:
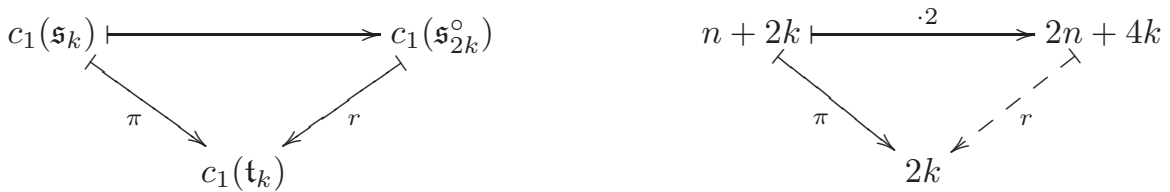

From this, we obtain that $\left.\mathfrak{s}_{2 k}^{\circ}\right|_{S_{n}^{3}(\bar{K})}=\mathfrak{t}_{k}$.

For the case of $\mathfrak{s}_{n+2 k}^{\circ}$, recall that $c_{1}\left(\mathfrak{s}_{n+2 k}^{\circ}\right)=4 n+4 k$. From the commutativity of the diagram below we deduce that $\left.\mathfrak{s}_{n+2 k}^{\circ}\right|_{S_{n}^{3}(\bar{K})}=\mathfrak{t}_{k}$.

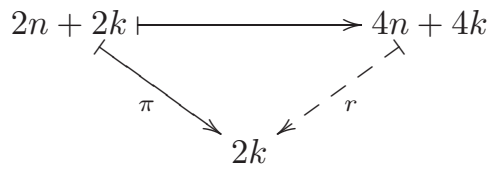

4.5. The case $e(\widehat{F})$ even. When $e(\widehat{F})$ is even, $H^{2}\left(W^{\circ}\right) \cong \mathbb{Z}^{h} \oplus \mathbb{Z} / 2 \mathbb{Z}$ by Lemma 4.1 One can check that $\operatorname{Spin}_{\text {tor }}^{c}\left(W^{\circ}\right)$ is an affine space over a submodule

$$
\mathbb{Z} \oplus \mathbb{Z} / 2 \mathbb{Z} \subseteq \mathbb{Z}^{h} \oplus \mathbb{Z} / 2 \mathbb{Z}
$$

where the $\mathbb{Z}$ summand is generated by a primitive element $x$. One can then define

$$
\mathfrak{s}_{k}^{\circ}:=\left.\mathfrak{s}_{k}\right|_{W^{\circ}} \in \operatorname{Spin}_{\text {tor }}^{c}\left(W^{\circ}\right)
$$

and, if $\gamma$ denotes the generator of the $\mathbb{Z} / 2 \mathbb{Z}$ summand,

$$
\widetilde{\mathfrak{s}_{k}^{\circ}}:=\mathfrak{s}_{k}^{\circ}+\gamma \in \operatorname{Spin}_{\text {tor }}^{c}\left(W^{\circ}\right) \text {. }
$$

One can check that $\widetilde{\mathfrak{s}_{k}^{\circ}}$ restricts to $Q$ to a non-extendible $\operatorname{spin}^{c}$ structure $\widetilde{\mathfrak{t}}$, and to $Y$ to the $\operatorname{spin}^{c}$ structure $\mathfrak{t}_{k+\frac{n}{2}}$. Moreover, we have that

$$
c_{1}\left(\widetilde{\mathfrak{s}_{k}^{\circ}}\right)^{2}=-\frac{(n+2 k)^{2}}{n} .
$$


Note that $n$ is even because so is $e(\widehat{F})$, so $k+\frac{n}{2}$ is an integer.

\section{A BOUND FOR THE NON-ORIENTABLE SLICE GENUS}

We now prove Theorem [1.1, that we restate here. Recall that we have defined $\varphi(K)$ to be the quantity $\min _{m \geq 0}\left\{m+2 V_{m}(\bar{K})\right\}$.

Theorem 1.1. For every knot $K$ in $S^{3}$,

$$
\gamma_{4}(K) \geq \frac{\sigma(K)}{2}-\varphi(K)
$$

Proof. Choose an odd integer $n>0$, and let $k$ be any integer. We denote by $[k]$ the representative for the residue class of $k$ modulo $n$ such that $0 \leq[k]<n$. By Remark 4.5 and Lemma 4.6, the $\operatorname{spin}^{c}$ structure $\mathfrak{s}_{n+2 k}^{\circ}$ restricts to a non-extendible $\operatorname{spin}^{c}$ structure on $Q$, that we denote by $\widetilde{\mathfrak{t}}$, and to $\mathfrak{t}_{k}$ on $Y$.

We apply Theorem 2.4 to the cobordism $\left(W^{\circ}, \mathfrak{s}_{n+2 k}^{\circ}\right)$ turned upside down, i.e. seen as a cobordism from $\left(-Y, \mathfrak{t}_{k}\right)$ to $(-Q, \widetilde{\mathfrak{t}})$ : the assumption that the map $H_{1}(Y ; \mathbb{Q}) \rightarrow H_{1}\left(W^{\circ} ; \mathbb{Q}\right)$ be injective is automatically satisfied, since $Y$ is a rational homology sphere. The inequality of Theorem 2.4 then reads as follows:

$$
c_{1}\left(\mathfrak{s}_{n+2 k}^{\circ}\right)^{2}+b_{2}^{-}\left(W^{\circ}\right) \leq 4 \underline{d}(-Q, \widetilde{\mathfrak{t}})+2 b_{1}(Q)-4 d\left(-S_{-n}^{3}(K), \mathfrak{t}_{k}\right) .
$$

We now compute each term of Equation (5.1). We have that $b_{2}^{-}\left(W^{\circ}\right)=1$ and $b_{1}(Q)=h-1$. Moreover,

$$
c_{1}\left(\mathfrak{s}_{n+2 k}^{\circ}\right)^{2}=((4 n+4 k) x)^{2}=-\frac{1}{4 n} \cdot(4 n+4 k)^{2}=-\frac{4}{n} \cdot(n+k)^{2},
$$

where we used the fact that $Q^{W^{\circ}}(2 x, 2 x)=-\frac{1}{n}$.

As for the $d$-invariant of $S_{-n}^{3}(K)$, by Theorems 2.2 and 2.3 we have

$$
d\left(-S_{-n}^{3}(K), \mathfrak{t}_{k}\right)=d\left(S_{n}^{3}(\bar{K}), \mathfrak{t}_{k}\right)=-\frac{n-(2[k]-n)^{2}}{4 n}-2 \max \left\{\bar{V}_{[k]}, \bar{V}_{n-[k]}\right\},
$$

where we set $\bar{V}_{i}:=V_{i}(\bar{K})$.

Finally, by [14, Theorem 5.1] and Theorem 2.2 above, we have that

$$
\underline{d}(-Q, \widetilde{\mathfrak{t}})=-d_{t}(Q, \widetilde{\mathfrak{t}})=-\left(\frac{e(\widehat{F})-2}{4}+a\right) \leq-\frac{e-n-2}{4} .
$$

Therefore, Equation (5.1) becomes

$$
-\frac{4}{n} \cdot(n+k)^{2}+1 \leq \frac{n-(2[k]-n)^{2}}{n}+8 \max \left\{\bar{V}_{[k]}, \bar{V}_{n-[k]}\right\}-(e-n-2)+2 h-2,
$$

which can be re-written as follows:

$$
2 h+8 \max \left\{\bar{V}_{[k]}, \bar{V}_{n-[k]}\right\} \geq e-n-\frac{4(n+k)^{2}-(2[k]-n)^{2}}{n}
$$

By combining it with Equation (3.2) as in [2, we obtain:

$$
4 h+8 \max \left\{\bar{V}_{[k]}, \bar{V}_{n-[k]}\right\} \geq 2 \sigma(K)-n-\frac{4(n+k)^{2}-(2[k]-n)^{2}}{n} .
$$

Given a fixed integer $m \geq 0$, it is not difficult to check that the best bound for $h$ coming from Equation (5.3) and involving $\bar{V}_{m}$ is obtained by setting $n=2 m+2 j+1$ and $k=-n \pm m$ (where $j$ is an arbitrary non-negative integer). The bound for $\gamma_{4}(K)$ that we obtain in this case is then:

$$
\gamma_{4}(K) \geq \frac{\sigma}{2}-m-2 \bar{V}_{m}
$$

By taking the maximum over $m \geq 0$ we conclude the proof of the theorem.

Remark 5.1. By setting $m=0$ in Equation (5.4), we obtain exactly Batson's inequality (1.1). 

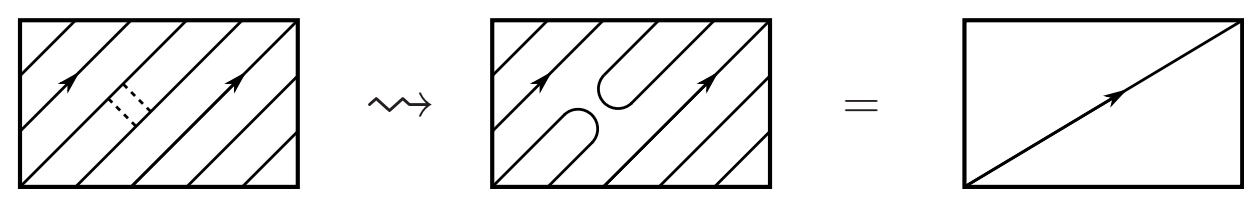

Figure 2. The figure shows a non-orientable cobordism of genus 1 from $T_{3,5}$ to the unknot. The rectangle above represents a torus, which is embedded in $S^{3}$ in the standard way. The (unoriented) band surgery above carries $T_{3,5}$ to the unknot.

Remark 5.2. For every $m \geq 0$, the bound in Equation (5.4) is sharp, in the sense that for each $m$ there exists a knot $K_{m}$ such that $\gamma_{4}\left(K_{m}\right)=\frac{\sigma(K)}{2}-m-V_{m}(\bar{K})$. The knot $K_{0}=T_{3,-4}$ exhibits that the inequality is sharp for $m=0$, as already shown by Batson [2].

For $m \geq 1$, consider the torus knot $K=T_{3,-5}$, whose signature is 8 . Since $\bar{K}=T_{3,5}$ is a positive torus knot, hence an L-space knot, the invariants $V_{i}\left(T_{3,5}\right)$ coincide with the torsion coefficients [19, Corollary 7.5]:

$$
V_{i}(\bar{K})=\sum_{j>0} j a_{j+i}
$$

where

$$
\Delta_{\bar{K}}(t)=a_{0}+\sum_{j>0} a_{j}\left(t^{j}+t^{-j}\right)
$$

is the Alexander polynomial of $\bar{K}$. One can explicitly compute that, for $\bar{K}=T_{3,5}$,

$$
\Delta_{T_{3,5}}(t)=t^{4}-t^{3}+t-1+t^{-1}-t^{-3}+t^{-4}
$$

It follows that $V_{1}(\bar{K})=1$ and that Equation (5.4) for $m=1$ gives

$$
\gamma_{4}(K) \geq \frac{8}{2}-(1+2)=1 \text {. }
$$

Since $\bar{K}$ bounds a Moebius band in $B^{4}$, as shown in Figure 2 (see also 2. Section 4]), it follows that (5.4) is sharp for $m=1$.

For all $m>1$, consider the knot $m K$, the connected sum of $m$ copies of $K$. Recall from [5, Proposition 6.1] that the sequence $\left\{V_{i}(K)\right\}$ satisfies the following subadditivity property: $V_{k+l}(K \# L) \leq$ $V_{k}(K) \# V_{l}(L)$ for each pair $(k, l)$ of non-negative integers and each pair $(K, L)$ of knots. By subadditivity of $\gamma_{4}$, subadditivity of the $V_{i}$, and additivity of the signature, we obtain

$$
\begin{aligned}
m=m \gamma_{4}(K) & \geq \gamma_{4}(m K) \\
& \geq \frac{\sigma(m K)}{2}-\left(m+2 V_{m}(m \bar{K})\right) \\
& \geq m\left(\frac{\sigma(K)}{2}-\left(1+2 V_{1}(\bar{K})\right)\right)=m .
\end{aligned}
$$

It follows that all the inequalities above are actually equalities, and that therefore (5.4) is sharp for every $m \geq 1$.

Remark 5.3. In the proof of Theorem 1.1 we only considered surgery with some odd framing $n>0$. If we considered the case of even $n$, and applied Theorem 2.4 to the torsion $\operatorname{spin}^{c}$ structure $\widetilde{\mathfrak{s}_{k}^{\circ}}$ (defined in Section 4.5), we would have obtained exactly the same bound as Equation (5.4) for all $m \geq 0$.

\section{Comparison to other Bounds}

In this section we study some properties of the functions $\varphi$ and $\omega$ defined in the introduction, and discuss the relationship between the bounds given by (1.1), (1.2), and (1.3).

Proposition 6.1. The invariant $\varphi$ is a concordance invariant, with values in the non-negative integers. It has the following properties: 
(1) $0 \leq \varphi(K) \leq \min \left\{\nu^{+}(\bar{K}), 2 V_{0}(\bar{K})\right\}$;

(2) $\varphi(K)=0$ if and only if $V_{0}(\bar{K})=\nu^{+}(\bar{K})=0$; in particular, if $K$ is slice, $\varphi(K)=0$;

(3) if there is an orientable genus- $g$ cobordism from $K_{1}$ to $K_{2}$, then $\left|\varphi\left(K_{1}\right)-\varphi\left(K_{2}\right)\right| \leq g$;

(4) if $K_{+}$is obtained from $K_{-}$by performing a crossing change from negative to positive, then $\varphi\left(K_{-}\right)-1 \leq \varphi\left(K_{+}\right) \leq \varphi\left(K_{-}\right)$;

(5) for every two knots $K_{1}, K_{2}, \varphi\left(K_{1} \# K_{2}\right) \leq \varphi\left(K_{1}\right)+\varphi\left(K_{2}\right)$.

We remark here that, in particular, $\varphi$, much like $\nu^{+}$, and by constrast with $\sigma$ and $v$, does not induce a homomorphism from the concordance group to the integers.

Proof. The sequence $\left\{V_{i}(K)\right\}_{i}$ is a concordance invariant, hence so is $\varphi$; moreover, the quantity $m+$ $2 V_{m}(\bar{K})$ is a non-negative integer for each $m$, and hence so is $\varphi(K)$.

(1) When $m=0, m+2 V_{m}(\bar{K})=2 V_{0}(\bar{K})$, while for $m=\nu^{+}(\bar{K}), m+2 V_{m}(\bar{K})=\nu^{+}(K)$. By definition, $\varphi(K) \leq 2 V_{0}(\bar{K})$ and $\varphi(K) \leq \nu^{+}(\bar{K})$.

(2) Observe that $m+2 V_{m}(\bar{K})$ is always strictly positive if $m>0$; hence, if $\varphi(K)=\min _{m}\{m+$ $\left.2 V_{m}(\bar{K})\right\}=0$, the minimum can only be attained at $m=0$, and in that case $V_{0}(\bar{K})=0$, which implies $\nu^{+}(\bar{K})=0$. The converse is obvious.

When $K$ is slice, $\nu^{+}(\bar{K})=0$, and hence $\varphi(K)$ vanishes, too.

(3) By [5, Lemma 5.1] we have that, under the given assumptions, $V_{m+g}\left(K_{1}\right) \leq V_{m}\left(K_{2}\right)$ for each non-negative integer $m$. It follows that $m+g+2 V_{m+g}\left(K_{1}\right) \leq m+2 V_{m}\left(K_{2}\right)+g$, hence, minimising over $m$,

$$
\varphi\left(K_{1}\right) \leq \min _{m^{\prime} \geq g}\left\{m^{\prime}+2 V_{m^{\prime}}\left(K_{2}\right)\right\} \leq \varphi\left(K_{2}\right)+g .
$$

Exchanging the roles of $K_{1}$ and $K_{2}$, we obtain the symmetric inequality.

(4) Observe that there is a genus- 1 cobordism from $K_{-}$to $K_{+}$, obtained by smoothing the double point in the trace of the crossing change homotopy. Thus, point (3) above shows that $\varphi\left(K_{-}\right)-$ $1 \leq \varphi\left(K_{+}\right)$. Using [7, Theorem 6.1] we also obtain:

$$
V_{m}\left(\overline{K_{+}}\right) \leq V_{m}\left(\overline{K_{-}}\right)
$$

from which, for each $m \geq 0$,

$$
m+2 V_{m}\left(\overline{K_{+}}\right) \leq m+2 V_{m}\left(\overline{K_{-}}\right),
$$

and minimising over all values of $m$ yields the desired inequality.

(5) For each $k, l$ non-negative integers, $V_{k+l}\left(K_{1} \# K_{2}\right) \leq V_{k}\left(K_{1}\right)+V_{l}\left(K_{2}\right)$ by [5. Proposition 6.1], hence

$$
\begin{aligned}
\varphi\left(K_{1} \# K_{2}\right) & =\min _{n}\left\{n+2 V_{n}\left(\overline{K_{1} \# K_{2}}\right)\right\} \\
& \leq \min _{n} \min _{k+l=n}\left\{k+l+2 V_{k}\left(\overline{K_{1}}\right)+2 V_{l}\left(\overline{K_{2}}\right)\right\} \\
& =\min _{k}\left\{k+2 V_{k}\left(\overline{K_{1}}\right)\right\}+\min _{l}\left\{l+2 V_{l}\left(\overline{K_{2}}\right)\right\} \\
& =\varphi\left(K_{1}\right)+\varphi\left(K_{2}\right) .
\end{aligned}
$$

We will compare our bound with (1.2) obtained by Ozsváth-Stipsicz-Szabó, and in order to do so we need to compare $v(K)$ with $\varphi(K)$. We say that a knot is Floer-thin if its knot Floer homology is supported on the diagonal $i-j=-\tau(K)$.

Proposition 6.2. When $K$ is a Floer-thin knot with $\tau(K) \geq 0$ or an L-space knot, then $\varphi(\bar{K})=$ $-v(K)$ and $\varphi(K)=0$.

In particular, the bound given by (5.4) for both $K$ and $\bar{K}$ is at most as strong as the one given by $v$, when $K$ is an L-space knot or an alternating knot. 
Proof. Recall that for a Floer-thin knot $K$ with $\tau(K)= \pm n$, we have $V_{i}(K)=V_{i}\left(T_{2, \pm(2 n+1)}\right)$ [1, Equation (8)], and hence $\varphi(K)=\varphi\left(T_{2, \pm(2 n+1)}\right)$. Analogously, it follows from [17, Theorem 1.14] that $v(K)=v\left(T_{2, \pm(2 n+1)}\right)$. It follows that it is enough to prove the statement for L-space knots.

When $K$ is an L-space knot, then a direct computation from the knot Floer complex shows that $V_{i}(\bar{K})=0$ for every $i$; hence $\varphi(K)=0$. On the other hand, Borodzik and Hedden have shown in 7 , Proposition 4.6] that

$$
v(K)=\Upsilon_{K}(1)=-\min _{n}\left\{n+2 V_{n}(K)\right\}=-\varphi(\bar{K}),
$$

as desired.

In the case of Floer-thin knots we can actually say more about $\varphi$.

Proposition 6.3. If $K$ is a Floer-thin knot with $\tau(K) \geq 0$, then we have

$$
\varphi(\bar{K})=\nu^{+}(K)=\tau(K)=-v(K) .
$$

If, additionally, $K$ is quasi-alternating, then $\varphi(\bar{K})=-\sigma(K) / 2$, and in this case the bounds (1.2) and (1.3) - applied to $K$ and $\bar{K}$ - yield

$$
\gamma_{4}(K) \geq 0 .
$$

Proof. By [1, Equation (8)], we know that the minimum of $\left\{m+2 V_{m}(K)\right\}$ is attained at $m=\tau(K)=$ $\nu^{+}(K)$. This implies at once that $\varphi(\bar{K})=\tau(K)$. The equality with $-v(K)$ follows from Proposition 6.2 .

When $K$ is quasi-alternating, $\tau(K)=-\sigma(K) / 2$, and the second part of the statement readily follows.

In many instances, the bound given by $v$ is better than the one given by $\varphi$; this is true, for example, for many knots of the form $K_{1} \# \overline{K_{2}}$, where $K_{1}$ and $K_{2}$ are L-space knots.

Example 6.4. Consider the two knots $K_{1}=T_{2,3}, K_{2}=T_{5,6}$, and let $K=K_{1} \# \bar{K}_{2}$. One computes $\sigma\left(K_{1}\right)=-2, \sigma\left(K_{2}\right)=-16, v\left(K_{1}\right)=-1$ and $v\left(K_{2}\right)=-6$. Using the techniques from [13] as in [5], we can also compute $\varphi(K)=6$ and $\varphi(\bar{K})=0$.

It follows that the bound given by (1.3), applied to both $K$ and $\bar{K}$, gives $\gamma_{4}(K) \geq 1$, while the bound given by (1.2) is $\gamma_{4}(K) \geq 2$.

As a consequence of Proposition 6.1 we deduce the following interesting feature of $\varphi$.

Corollary 6.5. The invariant $\varphi(K)$ is subadditive. In particular, the following identity holds:

$$
\lim _{n \rightarrow \infty} \frac{1}{n} \varphi(n K)=\inf _{n} \frac{1}{n} \varphi(n K) .
$$

Proof. By property (5) of Proposition 6.1 the function $n \mapsto \varphi(n K)$ is subadditive, in the sense that $\varphi(a K+b K) \leq \varphi(a K)+\varphi(b K)$ for every $a, b \geq 0$. The existence of the limit follows from Fekete's lemma [10.

Definition 6.6. We call $\omega(K)=\lim _{n} \frac{1}{n} \varphi(n K)$.

We now introduce the stable non-orientable 4 -genus $\gamma_{4}^{\text {st }}(K)$ of $K$, i.e. the $\operatorname{limit}_{\lim } \rightarrow \infty \frac{1}{n} \gamma_{4}(n K)$. Notice that the limit exists since the sequence $\left(\gamma_{4}(n K)\right)_{n}$ is subadditive, and that $\gamma_{4}^{\text {st }}(K) \leq \gamma_{4}(K)$.

Theorem 6.7. The invariant $\omega(K)$ is a concordance invariant of $K$, and it descends to a subadditive, homogeneous function $\omega: \mathcal{C} \rightarrow \mathbb{R}_{\geq 0}$. Additionally:

(1) $\gamma_{4}^{\text {st }}(K) \geq \frac{\sigma(K)}{2}-\omega(K)$;

(2) if there is an orientable genus- $g$ cobordism between $K_{1}$ and $K_{2}$, then $\left|\omega\left(K_{1}\right)-\omega\left(K_{2}\right)\right| \leq g$;

(3) if there is a crossing change (from negative to positive) from $K_{-}$to $K_{+}$, then $\omega\left(K_{-}\right)-1 \leq$ $\omega\left(K_{+}\right) \leq \omega\left(K_{-}\right)$.

As an immediate corollary to the theorem, we get the following:

Corollary 6.8. If the inequality in Theorem 1.1 is sharp, then $\gamma_{4}(n K)=n \gamma_{4}(K)$ for each $n$; in particular $\gamma_{4}^{\mathrm{st}}(K)=\gamma_{4}(K)$. 
As remarked for $\varphi$ above, $\omega$ is not a homomorphism, since it takes only non-negative values. Note also that $\omega$ is not identically 0 , since, by Proposition 6.3 applied to $n K$ for all $n \geq 0, \omega(K)$ coincides with $\sigma(K) / 2$ for Floer-thin knots with positive signature.

Also, by definition, $\omega(K) \leq \varphi(K)$, and in particular the bound for $\gamma_{4}^{\text {st }}(K)$ given by $\omega$ can be better than the bound given by $\varphi$ on $\gamma_{4}(K)$ (see Proposition 7.1 for an example). This is by contrast with the bound given, for example, by $\tau, s$, or $\nu^{+}$on the stable orientable slice genus: the first two are linear, while the third is sublinear in $K$ [5, Theorem 1.4].

Proof of Theorem 6.7. The invariant $\omega$ is a concordance invariant, since $\varphi$ is, and it takes non-negative values, since $\varphi$ does. Moreover, it is subadditive by construction:

$$
\begin{aligned}
\omega(K \# L) & =\lim _{n}\left\{\frac{1}{n} \varphi(n(K \# L))\right\} \leq \lim _{n}\left\{\frac{1}{n}(\varphi(n K)+\varphi(n L))\right\}= \\
& =\lim _{n}\left\{\frac{1}{n} \varphi(n K)\right\}+\lim _{n}\left\{\frac{1}{n} \varphi(n L)\right\}=\omega(K)+\omega(L),
\end{aligned}
$$

where the inequality follows from the subadditivity of $\varphi$ (Property (5) of Proposition 6.1).

It is also homogeneous, in the sense that $\omega(n K)=n \omega(K)$ :

$$
\omega(n K)=\lim _{m} \frac{1}{m} \varphi(m n K)=n \lim _{m} \frac{1}{m n} \varphi(m n K)=n \lim _{m^{\prime}} \frac{1}{m^{\prime}} \varphi\left(m^{\prime} K\right)=n \omega(K) .
$$

(1) Applying (5.4) for $n K$ we obtain, for each $n \geq 1$ :

from which

$$
\gamma_{4}(n K) \geq \frac{\sigma(n K)}{2}-\varphi(n K)=n \frac{\sigma(K)}{2}-\varphi(n K),
$$

$$
\gamma_{4}^{\text {st }}(K)=\lim _{n} \frac{\gamma_{4}(n K)}{n} \geq \frac{\sigma(K)}{2}-\lim _{n} \frac{\varphi(n K)}{n}=\frac{\sigma(K)}{2}-\omega(K) .
$$

Properties (2) and (3) follow immediately from the corresponding properties of $\varphi$, stated in Proposition 6.1 above.

\section{An example}

An interesting feature of $\omega$ is that - by contrast with $\varphi$ - it can attain non-integer values, as we shall see presently.

To this end, we study an example in detail: we show that $\omega\left(T_{2,3}-T_{5,6}\right)=\frac{26}{5}$. Before doing so, we recall some facts about Krcatovich's reduced knot Floer complex.

In [13, Krcatovich associates to each knot $J \subset S^{3}$ a reduced version of the knot Floer complex, denoted by $\underline{\mathrm{CFK}}^{-}(J)$. The reduced knot Floer complex for L-space knots is of a particularly simple form, in that it only consists of a single tower, i.e. it is isomorphic to $\mathbb{F}[U]$ as an $\mathbb{F}[U]$-module, but not as a graded module (see [13, Corollary 4.2]).

Krcatovich also observed that, if one is only concerned with correction terms, the connected sum of two L-space knots behaves as an L-space knot [13, Example 2]; more specifically, he showed that if $K$ and $K^{\prime}$ are L-space knots, then $\underline{\mathrm{CFK}}^{-}\left(K \# K^{\prime}\right)$ fits in a short exact sequence of complexes:

$$
0 \rightarrow T \rightarrow \underline{\mathrm{CFK}}^{-}\left(K \# K^{\prime}\right) \rightarrow A \rightarrow 0,
$$

where $T$ is a tower and $A$ is acyclic. In this case, we will write $\underline{\mathrm{CFK}}^{-}\left(K \# K^{\prime}\right) \approx T$; moreover, if $C$ is another chain complex such that $C \approx T$, we will also write $\underline{\mathrm{CFK}^{-}}\left(K \# K^{\prime}\right) \approx C$. In Krcatovich's terminology, $\underline{\mathrm{CFK}}^{-}\left(K \# K^{\prime}\right)$ has a representative staircase, which is determined by $T$; conversely, the staircase determines $T$ and the collection $\left\{V_{i}\left(K \# K^{\prime}\right)\right\}$. Moreover, for any other knot $L$, we can use $T$ as a substitute for $\underline{\mathrm{CFK}}^{-}\left(K \# K^{\prime}\right)$ to compute $\mathrm{CFK}^{-}\left(K \# K^{\prime} \# L\right)$, in the sense that there is a filtered quasi-isomorphism

$$
T \otimes \mathrm{CFK}^{-}(L) \cong \underline{\mathrm{CFK}}^{-}\left(K \# K^{\prime}\right) \otimes \mathrm{CFK}^{-}(L) .
$$

Proposition 7.1. Let $K=T_{2,3}-T_{5,6}$. Then $\omega(K)=\frac{26}{5}<\varphi(K)=6$. Moreover, $\omega(K)<\frac{\varphi(n K)}{n}$ for all $n \in \mathbb{Z}_{>0}$, so the limit in Definition $[6.6$ is not attained at any $n$. 
Before proving the proposition, recall that it is proven in [6] that, in the case of torus knots $T_{p, q}$, the representative staircase is determined by the arithmetics of $p$ and $q$ (compare also with [8, Section $5]$ ). In what follows, we will be concerned with the connected sum $n T_{5,6}$ of $n$ copies of $T_{5,6}$, and in this case the result reads:

$$
\underline{\mathrm{CFK}}^{-}\left(n T_{5,6}\right) \approx \underline{\mathrm{CFK}}^{-}\left(T_{5,5 n+1}\right) .
$$

That is, the representative staircase for $n T_{5,6}$ is the staircase of $T_{5,5 n+1}$.

We will also need a lemma about $n T_{2,3}$. This is true in wider generality (see [6]), but we prove it here in a special case.

Lemma 7.2. For each positive integer $n$, the complex $\mathrm{CFK}^{\infty}\left( \pm n T_{2,3}\right)$ is filtered chain homotopy equivalent to $\operatorname{CFK}^{\infty}\left( \pm T_{2,2 n+1}\right) \oplus A_{ \pm n}$, where $A_{ \pm n}$ is an acyclic complex over $\mathbb{F}[U]$.

Proof. It suffices to prove the statement for $\operatorname{CFK}^{\infty}\left(n T_{2,3}\right)$, since the corresponding statement for $\operatorname{CFK}^{\infty}\left(-n T_{2,3}\right)$ follows by taking duals: in fact, $\operatorname{CFK}^{\infty}(\bar{K})$ is isomorphic to the dual of $\mathrm{CFK}^{\infty}(K)$, and taking duals preserves direct sums and acyclicity.

We will now prove the statement for $\operatorname{CFK}^{\infty}\left(n T_{2,3}\right)$ by induction on $n$ : recall that $\operatorname{CFK}^{\infty}\left((n+1) T_{2,3}\right)$ is filtered quasi-isomorphic to $\mathrm{CFK}^{\infty}\left(n T_{2,3}\right) \otimes \mathrm{CFK}^{\infty}\left(T_{2,3}\right)$, and that $\mathrm{CFK}^{\infty}\left(T_{2,3}\right)$ is filtered quasiisomorphic to $\left(\mathbb{F}\left[U, U^{-1}\right] a \oplus \mathbb{F}\left[U, U^{-1}\right] b \oplus \mathbb{F}\left[U, U^{-1}\right] c, \partial_{1}\right)$, where $\partial_{1} b=U a+c$ and $a$ and $c$ are cycles; moreover, the Alexander gradings of the generators are $A(a)=1, A(b)=0, A(c)=-1$.

By induction, we can assume that $\operatorname{CFK}^{\infty}\left(n T_{2,3}\right)=\operatorname{CFK}^{\infty}\left(T_{2,2 n+1}\right) \oplus A_{n}$, where $\operatorname{CFK}^{\infty}\left(T_{2,2 n+1}\right)$ is generated over $\mathbb{F}\left[U, U^{-1}\right]$ by $x_{1}, \ldots, x_{2 n+1}$, is equipped with the differential $\partial_{n}$ defined by

$$
\partial_{n} x_{2 i}=U x_{2 i-1}+x_{2 i+1}, \quad \partial_{n} x_{2 i+1}=0,
$$

and the Alexander grading is $A\left(x_{i}\right)=n+1-i$.

We observe that, whenever $A$ is acyclic, $A \otimes C$ is acyclic for every other complex $C$. Therefore, in order to prove the theorem, it suffices to show that $\operatorname{CFK}^{\infty}\left(T_{2,2 n+1}\right) \otimes \operatorname{CFK}^{\infty}\left(T_{2,3}\right) \cong \operatorname{CFK}^{\infty}\left(T_{2,2 n+3}\right) \oplus$ $A$, where $A$ is acyclic.

To this end, consider the subspace $V$ of $\mathrm{CFK}^{\infty}\left(T_{2,2 n+1}\right) \otimes \mathrm{CFK}^{\infty}\left(T_{2,3}\right)$ spanned by:

$$
V=\operatorname{Span}_{\mathbb{F}\left[U, U^{-1}\right]}\left\{x_{1} a, x_{1} b, x_{i} c\right\},
$$

where we drop the $\otimes$ between generators to ease readability, so that $x_{1} a$ really means $x_{1} \otimes a$. It is easy to check that $V$ is in fact a subcomplex of $\operatorname{CFK}^{\infty}\left(T_{2,2 n+1}\right) \otimes \operatorname{CFK}^{\infty}\left(T_{2,3}\right)$, and that $V$ is indeed isomorphic to $\mathrm{CFK}^{\infty}\left(T_{2,2 n+3}\right)$. In fact, an explicit isomorphism is given by $x_{1} a \mapsto x_{1}, x_{1} b \mapsto x_{2}, x_{i} c \mapsto x_{i+2}$.

We claim that $V$ has a complement, which is the direct sum of copies of rank-4 subspaces $W_{2 i}$, for $i=1, \ldots, n$.

$$
W_{2 i}=\operatorname{Span}_{\mathbb{F}\left[U, U^{-1}\right]}\left\{x_{2 i} b, x_{2 i-1} b+x_{2 i} a, x_{2 i+1} b+x_{2 i} c, x_{2 i+1} c\right\} .
$$

It is easy to prove that $W_{2 i}$ is in fact an acyclic subcomplex for each $i$, and that the $W_{2 i}$ together with $V$ span all of $\mathrm{CFK}^{\infty}\left(T_{2,2 n+1}\right) \otimes \mathrm{CFK}^{\infty}\left(T_{2,3}\right)$.

Moreover, since the ranks of $V$ and $W_{2 i}$ add up to the rank of $\operatorname{CFK}^{\infty}\left(T_{2,2 n+1}\right) \otimes \operatorname{CFK}^{\infty}\left(T_{2,3}\right)$, this is actually a direct sum decomposition of complexes. Since the $W_{2 i}$ are acyclic, we have exhibited the desired decomposition.

We can now turn to the proof of Proposition 7.1 .

Proof of Proposition 7.1. Let $K_{1}=T_{2,3}$ and $K_{2}=T_{5,6}, K=K_{1}-K_{2}$. The fact that $\varphi(K)=6$ was already observed in Example 6.4. Let now $L_{n}=n K=n K_{1}-n K_{2}$, and $n=5 \ell$. We will prove that for $\ell \in \mathbb{Z}_{>0}$ we have

$$
\varphi\left(L_{5 \ell}\right)=26 \ell+1 .
$$

This implies at once that $\omega(K)=\lim _{n} \frac{\varphi\left(L_{n}\right)}{n}=\frac{26}{5}$, and that $\varphi\left(L_{5 \ell}\right)>\omega(K) \cdot 5 \ell$ for each $\ell$. Moreover, by definition, for each $n$

$$
\varphi\left(L_{n}\right) \geq \frac{26}{5} n
$$


for all $n \in \mathbb{Z}_{>0}$; since right-hand side is an integer only if $n$ is a multiple of 5 , the inequality is strict also for all $n$ not divisible by 5 , hence the limit is never attained.

We now set out to prove that $\varphi\left(L_{5 \ell}\right)=26 \ell+1$.

Since $\mathrm{CFK}^{-}\left(n K_{2}\right) \approx \mathrm{CFK}^{-}\left(T_{5,5 n+1}\right)$, we can use Lemma 7.2 and results from [5] to compute the invariants $V_{i}\left(n K_{2}-n K_{1}\right)$, treating $n K_{2}$ as $T_{5,5 n+1}$ and $-n K_{1}$ as $-T_{2,2 n+1}$. Indeed, let $J_{i}=5 \ell K_{i}$ for $i=1,2$.

Given a semigroup $\Gamma \subseteq \mathbb{N}=\{0,1, \ldots\}$, we denote by $\Gamma(\cdot)$ its enumerating function, i.e. the unique strictly increasing function

$$
\Gamma: \mathbb{N} \rightarrow \mathbb{N}
$$

which is surjective on $\Gamma$. Note that $\Gamma(0)=0$. Given an integer $x$, we denote $(x)_{+}=\max \{0, x\}$. Since $\mathrm{CFK}^{\infty}\left(-n T_{2,3}\right)$ is, up to an acyclic summand, $\mathrm{CFK}^{\infty}\left(-T_{2,2 n+1}\right)$, we can apply [5, Theorem 3.1 and Remark 3.3] and obtain:

$$
\nu_{v}^{+}(5 \ell \bar{K}):=\min \left\{i \mid V_{i}(5 \ell \bar{K}) \leq v\right\}=\left(\max _{k \geq 0}\left\{g\left(J_{2}\right)-g\left(J_{1}\right)+\Gamma_{J_{1}}(k)-\Gamma_{J_{2}}(k+v)\right\}\right)_{+},
$$

where $\Gamma_{J_{1}}(\cdot)$ and $\Gamma_{J_{2}}(\cdot)$ are the enumerating functions associated to the semigroups

$$
\Gamma_{J_{1}}=\langle 2,10 \ell+1\rangle ; \quad \Gamma_{J_{2}}=\langle 5,25 \ell+1\rangle .
$$

The genera of the knots $J_{1}$ and $J_{2}$ are respectively $5 \ell$ and $50 \ell$, so the formula for $\nu_{v}^{+}$becomes

$$
\nu_{v}^{+}\left(\overline{L_{5 \ell}}\right)=\left(45 \ell-\min _{k \geq 0}\left\{\Gamma_{J_{2}}(k+v)-\Gamma_{J_{1}}(k)\right\}\right)_{+} .
$$

Note that, with this notation, we have that

$$
\varphi\left(L_{5 \ell}\right)=\min _{v \geq 0}\left\{\nu_{v}^{+}\left(\overline{L_{5 \ell}}\right)+2 v\right\},
$$

which we are now going to compute.

The enumerating functions above can be expressed in the following equations:

$$
\begin{gathered}
\Gamma_{J_{1}}(k)= \begin{cases}2 k & 0 \leq k \leq 5 \ell \\
5 \ell+k & k \geq 5 \ell\end{cases} \\
\Gamma_{J_{2}}(k)= \begin{cases}5 k & 0 \leq k \leq 5 \ell \\
25 \ell+5\left\lfloor\frac{k-5 \ell}{2}\right\rfloor+[k-5 \ell]_{2} & 5 \ell \leq k \leq 15 \ell \\
50 \ell+5\left\lfloor\frac{k-15 \ell}{3}\right\rfloor+[k-15 \ell]_{3} & 15 \ell \leq k \leq 30 \ell \\
75 \ell+5\left\lfloor\frac{k-30 \ell}{4}\right\rfloor+[k-30 \ell]_{4} & 30 \ell \leq k \leq 50 \ell \\
50 \ell+k & k \geq 50 \ell\end{cases}
\end{gathered}
$$

Note that in Equation (7.1) we can in fact take the minimum over $0 \leq k \leq 5 \ell$, because for $k \geq 5 \ell$ the function $\Gamma_{J_{1}}(k)$ increases at a lesser or equal rate than any translate of $\Gamma_{J_{2}}$ : specifically, $\Gamma_{J_{1}}(k+j)-\Gamma_{J_{1}}(k)=j \leq \Gamma_{J_{2}}(k+v+j)-\Gamma_{J_{2}}(k+v)$. Therefore

$$
\nu_{v}^{+}\left(\overline{L_{5 \ell}}\right)=\left(45 \ell-\min _{0 \leq k \leq 5 \ell}\left\{\Gamma_{J_{2}}(k+v)-\Gamma_{J_{1}}(k)\right\}\right)_{+} .
$$

Now we return to the proof of Proposition 7.1. Recall that we want to prove that $\varphi(5 \ell K)=26 \ell+1$. By (7.2) we have

$$
\varphi\left(L_{5 \ell}\right)=\min _{v \geq 0}\left\{\nu_{v}^{+}\left(\overline{L_{5 \ell}}\right)+2 v\right\} .
$$

As shown in Lemma 7.3 below, the choice $v=13 \ell$ gives $\nu_{v}^{+}\left(\overline{L_{5 \ell}}\right)+2 v=26 \ell+1$. Moreover, it also follows from Lemma 7.3 that $V_{0}\left(\overline{L_{5 \ell}}\right)=13 \ell+1$, hence choosing $v \geq 13 \ell+1$ yields $2 v \geq 26 \ell+2>26 \ell+1$.

We now distinguish between $v \leq 5 \ell-1$ and $v \geq 5 \ell$. By Lemma 7.4 below, for $v \in[0,5 \ell-1]$ we have

$$
\nu_{v}^{+}\left(\overline{L_{5 \ell}}\right)+2 v=45 \ell-3 v \geq 45 \ell-15 \ell+3>26 \ell+1 ;
$$


by Lemma 7.5, on the other hand, for $v \in[5 \ell, 13 \ell-1]$ we have

$$
\nu_{v}^{+}\left(\overline{L_{5 \ell}}\right)+2 v \geq 2(13 \ell-v)+1+2 v=26 \ell+1 .
$$

This shows that $\varphi\left(L_{5 \ell}\right)=26 \ell+1$, as desired.

Lemma 7.3. $\nu_{13 \ell}^{+}\left(\overline{L_{5 \ell}}\right)=1$.

Proof. Note that, since $k \leq 5 \ell, k+13 \ell \in[13 \ell, 18 \ell]$. Therefore, the difference of the enumerating functions is

$$
f(k):=\Gamma_{J_{2}}(k+13 \ell)-\Gamma_{J_{1}}(k)= \begin{cases}45 \ell+5\left\lfloor\frac{k}{2}\right\rfloor+[k]_{2}-2 k & 0 \leq k \leq 2 \ell \\ 50 \ell+5\left\lfloor\frac{k-2 \ell}{3}\right\rfloor+[k-2 \ell]_{3}-2 k & 2 \ell \leq k \leq 5 \ell\end{cases}
$$

In the first interval $f(k+2) \geq f(k)$, while in the second interval $f(k+3) \leq f(k)$. It follows that the minimum is attained for some $k \in\{0,1,5 \ell-2,5 \ell-1,5 \ell\}$. A direct computation for these five values shows that the minimum is $45 \ell-1$, attained both at $k=1$ and at $k=5 \ell-1$. It follows that $\nu_{13 \ell}^{+}\left(\overline{L_{5 \ell}}\right)=45 \ell-(45 \ell-1)=1$.

Lemma 7.4. For each $v=0, \ldots, 5 \ell-1, \nu_{v}^{+}\left(\overline{L_{5 \ell}}\right)=45 \ell-5 v$.

Proof. Note that, since we only need to test $k \leq 5 \ell$ when computing the minimum in (7.1), we can assume that for each value of $v$ in the statement $k+v \leq 10 \ell-1$. Therefore, the difference of the enumerating functions is

$$
f(k):=\Gamma_{J_{2}}(k+v)-\Gamma_{J_{1}}(k)= \begin{cases}5 v+3 k & 0 \leq k \leq 5 \ell-v \\ 25 \ell+5\left\lfloor\frac{k+v-5 \ell}{2}\right\rfloor+[k+v-5 \ell]_{2}-2 k & 5 \ell-v \leq k \leq 5 \ell .\end{cases}
$$

Such a function is increasing on the interval $0 \leq k \leq 5 \ell-v$, and on the second interval it satisfies the condition $f(k+2)-f(k) \geq 1$. It follows that the minimum is attained for some $k=0,5 \ell-v$ or $5 \ell-v+1$. A direct computation for these values shows that the minimum is $5 v$, attained at $k=0$. Therefore, $\nu_{v}^{+}\left(\overline{L_{5 \ell}}\right)=45 \ell-5 v$.

Lemma 7.5. Let $v=13 \ell-s$ for some $0<s \leq 8 \ell$. Then $\nu_{v}^{+}\left(\overline{L_{5 \ell}}\right) \geq 2 s+1$.

Proof. Choosing $k=0$ in Equation (7.1), we obtain:

$$
\nu_{v}^{+}\left(\overline{L_{5 \ell}}\right) \geq 45 \ell-\Gamma_{J_{2}}(13 \ell-s)
$$

Since $13 \ell-s \in[5 \ell, 13 \ell] \subseteq[5 \ell, 15 \ell]$, we have

$$
\Gamma_{J_{2}}(13 \ell-s)=45 \ell+5\left\lfloor-\frac{s}{2}\right\rfloor+[s]_{2} .
$$

If $s \geq 2$ is even, then $\Gamma_{J_{2}}(13 \ell-s)=45 \ell-\frac{5}{2} s \leq 45 \ell-2 s-1$. If $s$ is odd, then $\Gamma_{J_{2}}(13 \ell-s)=$ $45 \ell-\frac{5}{2}(s+1)+1 \leq 45 \ell-2 s-1$. In both cases we have $\Gamma_{J_{2}}(13 \ell-s) \leq 45 \ell-2 s-1$, so we obtain

$$
\nu_{v}^{+}\left(\overline{L_{5 \ell}}\right) \geq 45 \ell-\Gamma_{J_{2}}(13 \ell-s) \geq 2 s+1 \text {. }
$$

With techniques similar to the ones used in Proposition 7.1 one can to show that $\omega$ attains many other positive non-integer values. We conclude with two questions concerning the image of $\omega$.

Question 7.6. Is $\mathbb{Q}_{\geq 0} \subseteq \operatorname{im}(\omega)$ ? Can $\omega$ take irrational values?

\section{REFERENCES}

1. Paolo Aceto and Marco Golla, Dehn surgeries and rational homology balls, to appear in Alg. Geom. Topol., 2015.

2. Joshua Batson, Nonorientable four-ball genus can be arbitrarily large, preprint arXiv:1204.1985, 2012.

3. _ Obstructions to slicing knots and splitting links, Ph.D. thesis, MIT, 2014.

4. Stefan Behrens and Marco Golla, Heegaard Floer correction terms, with a twist, preprint arXiv:1505.07401 2015.

5. Jozséf Bodnár, Daniele Celoria, and Marco Golla, A note on cobordisms of algebraic knots, preprint arXiv:1509.08821 2015.

6. Jozséf Bodnár and Marco Golla, in preparation.

7. Maciej Borodzik and Matthew Hedden, The $v$ function of L-space knots is a Legendre transform, preprint arXiv:1505.06672, 2015. 
8. Maciej Borodzik and Charles Livingston, Heegaard Floer homology and rational cuspidal curves, Forum Math. Sigma 2 (2014), e28, 23 pp.

9. Florian Deloup and Gwénaël Massuyeau, Quadratic functions and complex spin structures on three-manifolds, Topology 44 (2005), no. 3, 509-555.

10. Mihály Fekete, Über die Verteilung der Wurzeln bei gewissen algebraischen Gleichungen mit ganzzahligen Koeffizienten, Math. Z. 17 (1923), no. 1, 228-249.

11. Patrick M. Gilmer and Charles Livingston, The nonorientable 4-genus of knots, J. London Math. Soc. 84 (2011), no. 3, 559-577.

12. Jennifer Hom and Zhongtao Wu, Four-ball genus and a refinement of the Ozsváth-Szabó tau-invariant, to appear in J. Symp. Geom., 2014.

13. David Krcatovich, The reduced knot Floer complex, Topology and its applications 194 (2015), 171-201.

14. Adam S. Levine, Daniel Ruberman, and Sašo Strle, Non-orientable surfaces in homology cobordisms, Geom. Topol. 19 (2015), no. 1, 439-494, with an appendix by Ira M. Gessel.

15. Tye Lidman, On the infinity flavor of Heegaard Floer homology and the integral cohomology ring, Comment. Math. Helv. 88 (2013), no. 4, 875-898.

16. Yi Ni and Zhongtao Wu, Cosmetic surgeries on knots in $S^{3}$, J. Reine Angew. Math. 2015 (2015), no. 706, 1-17.

17. Peter S. Ozsváth, András I. Stipsicz, and Zoltán Szabó, Concordance homomorphisms from knot Floer homology, preprint arXiv:1407.1795, 2014.

18. _ Unoriented knot Floer homology and the unoriented four-ball genus, preprint arXiv:1508.03243 2015.

19. Peter S. Ozsváth and Zoltán Szabó, Absolutely graded Floer homologies and intersection forms for four-manifolds with boundary, Advances in Mathematics 173 (2003), no. 2, 179-261.

20. _ Knot Floer homology and integer surgeries, Algebraic \& Geometric Topology 8 (2008), no. 1, 101-153.

21. Jacob A. Rasmussen, Lens space surgeries and a conjecture of Goda and Teragaito, Geometry \& Topology 8 (2004), no. 3, 1013-1031.

Mathematical Institute, University of Uppsala, Box 480, 75106 Uppsala, Sweden

E-mail address: marco.golla@math.uu.se

Department of Mathematics, Imperial College London, 180 Queen's Gate, London SW7 2AZ, UK

E-mail address: m.marengon13@imperial.ac.uk 